\title{
Using Ambulatory Syndromic Surveillance Data for Chronic Disease: A BMI Case Study
}

\author{
Andrew Walsh* \\ Health Monitoring Systems, Inc, Pittsburgh, PA, USA
}

\section{Objective}

To demonstrate the utility of ambulatory syndromic surveillance data to public health domains beyond communicable diseases

\section{Introduction}

Syndromic surveillance is one of the meaningful use public health menu set objectives for eligible professionals. The value of this data for syndromic surveillance as an adjunct to the more widely adopted emergency department registrations has not been studied extensively. It may be that it would improve the sensitivity or timeliness of detecting certain communicable disease events, or it may just contain signals comparable to what is available via other syndromic surveillance data streams. The value of making the effort to collect this data is considered contingent on the answer to that question.

Public health is concerned with more than just communicable diseases, however. Chronic diseases and their underlying causes are also a significant public health concern. Obesity alone is estimated to be responsible for $2.5 \%$ of the global disease burden, and represents a higher fraction in many developed nations. Since chronic diseases are not associated with singular events of brief duration, they are difficult to track with traditional surveillance methods. They are also not typically managed via emergency departments, so syndromic surveillance does not capture them well either.

Chronic diseases are often treated by physicians at ambulatory practices. Thus data from eligible professionals may provide a means for monitoring chronic diseases, or metrics associated with chronic diseases, that would not otherwise be as feasible. As a proof of concept, this study seeks to determine if body mass index (BMI), the standard measure of obesity, can be obtained from ambulatory syndromic surveillance messages.

\section{Methods}

Syndromic surveillance messages were collected from approximately 100 eligible professionals at 21 practices in the greater Philadelphia metropolitan area over a period of 1 month. These were received and processed by the EpiCenter syndromic surveillance system. Height and weight were extracted from OBX segments and used to calculate BMI.

The study population was restricted to adults, as determined by visit date relative to the patient's reported date of birth. The data were also filtered to exclude outlier height and weight values, as determined by the distribution of these values in the dataset. These extreme values appeared to represent data entry errors. The mean height and weight for each patient were then calculated from all remaining observations, and those means were used to calculate the patient BMI.

\section{Results}

A total of 194,523 messages were received from 21 practices over the 1 month study period. These represented 24,076 unique visits from 18,858 patients, as determined by the identifiers sent by the practices. Of these, 18,047 were adults (95.7\%). Of those, 18,022 (99.9\%) had height and weight measurements determined to be within the limits determined from the distributions.
BMI was calculated for those 18,022 patients. It ranged from 10.5 to 122.7. The median BMI was 27.1 and the mean was 28.1. The full distribution of BMI is shown in Figure 1. 270 patients (1.5\%) had an underweight BMI, 6,194 patients (34.4\%) had an overweight BMI, and $5,679(31.5 \%)$ had an obese BMI.

\section{Conclusions}

This study successfully demonstrated that BMI can be extracted from ambulatory syndromic surveillance messages. Minimal data processing was necessary to achieve high data quality. Expanding to include children would make it more difficult to set lower limits for filtering errant height and weight values, but in this setting that filtering did not substantially affect the results. Taking the mode of values from a given visit rather than the mean might provide an alternative method to exclude some errant values.

A longer, longitudinal study would be necessary to determine whether monitoring patient BMI in this fashion could actually be used to detect meaningful trends of interest to public health, or used to improve significant public health outcomes.

Fig. 1: Histogram of Patient BMI

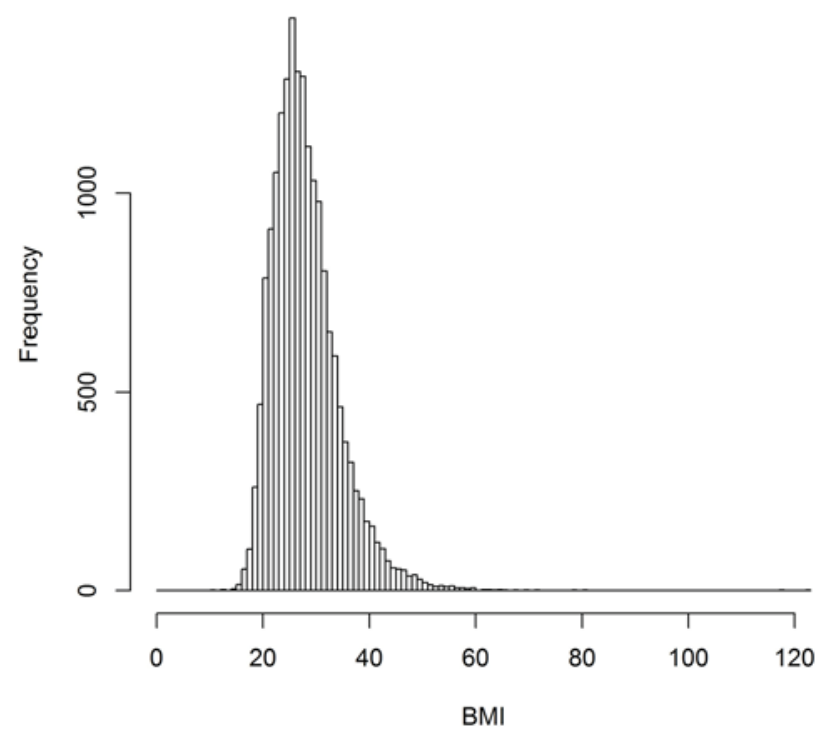

Keywords

Ambulatory data; Chronic disease surveillance; BMI; Syndromic surveillance

\section{Acknowledgments}

We wish to thank the Pennsylvania Department of Health for funding support and data for this work.

*Andrew Walsh

E-mail: andy.walsh@hmsinc.com 